\title{
Incretin mimetics as a novel therapeutic option for hepatic steatosis
}

Tushuizen ME, Bunck MC, Pouwels PJ, van Waesberghe JHT, Diamant M, Heine RJ. Incretin mimetics as a novel therapeutic option for hepatic steatosis.

Liver International 2006: 26: 1015-1017

(C) 2006 The Authors. Journal compilation (C) 2006 Blackwell Munksgaard

Abstract: Background: Fat accumulation in the liver or non-alcoholic fatty liver disease (NAFLD) is regarded as a key pathogenic factor and component of the metabolic syndrome. It was reported that administration of the incretin mimetic exenatide reversed hepatic steatosis in an obese mouse model. We had the opportunity to study the effect of additional exenatide administration on liver fat content in a patient with type 2 diabetes. Case report: A 59-yearold male with poorly controlled type 2 diabetes was treated with exenatide in addition to metformin monotherapy. Following 44 weeks of exenatide therapy, mean the liver fat measured by liver spectroscopy declined from $15.8 \%$ to $4.3 \%$. This dramatic decrease in liver fat was accompanied by significant beneficial changes in several cardiovascular disease risk factors and improvement of all liver enzymes, in particular alanine aminotransferase, the most important marker of liver steatosis. Conclusion: This case report suggests that the incretin mimetic exenatide decreases hepatic fat accumulation and may play a role in the future treatment of NAFLD, and the associated insulin resistance and cardiovascular risk factors in an evergrowing high-risk population.

\author{
Maarten E. Tushuizen', Mathijs C. \\ Bunck ${ }^{1}$, Petra J. Pouwels ${ }^{2}$, Jan Hein \\ T. van Waesberghe ${ }^{3}$, Michaela \\ Diamant $^{1}$ and Robert J. Heine ${ }^{1}$
}

Departments of ${ }^{1}$ Endocrinology/Diabetes Centre, ${ }^{2}$ Physics \& Medical Technology and ${ }^{3}$ Radiology, VU University Medical Centre, Amsterdam, The Netherlands
Key words: hepatic steatosis - incretin mimetics - metabolic syndrome - type 2 diabetes Maarten E. Tushuizen, MD, Department of Endocrinology/Diabetes Centre, VU University Medical Centre, PO Box 7057, 1007 MB Amsterdam, The Netherlands.

Tel: +31204443597

Fax: +31204440530

e-mail: m.tushuizen@vumc.nl

Received 18 May 2006, accepted 29 May 2006
The metabolic syndrome, a cluster of cardiovascular disease factors, is present in the majority of obese type 2 diabetic patients (1). Currently, fat accumulation in the liver or non-alcoholic fatty liver disease (NAFLD) is regarded as a key pathogenic factor and component of this syndrome (2). Therapies aimed at reducing liver fat have been shown to concomitantly improve the risk profile in this high-risk population. For example, both lifestyle interventions resulting in weight loss and administration of thiazolidinediones, which is associated with weight gain, have been associated with decreased liver fat and improvement of (hepatic) insulin sensitivity (3, 4). Furthermore, these studies suggested that the presence of risk factors for cardiovascular disease, in particular low HDL cholesterol and high triglycerides, is strongly related to the amount of liver fat.

Glucagon-like peptide 1 (GLP-1) is an incretin hormone that is secreted by the $\mathrm{L}$ cells of the intestine upon meal ingestion. In type 2 diabetic patients, GLP-1 lowers blood glucose levels in a glucose-dependent manner, by potentiating mealinduced insulin production and secretion by the pancreatic $\beta$ cells, by slowing gastric emptying and by inhibiting glucagon secretion. Also, GLP1 induces weight loss, possibly because of central inhibition of appetite, and may also improve insulin sensitivity (5). However, GLP-1 has a very short half-life as it is degraded by the ubiquitous enzyme dipeptidyl-peptidase IV (DPPIV). The long-acting, DPPIV-resistant GLP-1 receptor agonist or incretin mimetic exendin-4 (exenatide, synthetic exendin-4) is a 39 amino-acid peptide that binds with high affinity to the GLP receptor. Recently, exenatide was introduced, to the market in the United States as a blood-glucose-lowering-agent. The full scope of the effects of incretin mimetics still needs to be elucidated and the almost ubiquitous presence of the GLP-1 receptor in the human body implies many potential actions. Recently, it was reported that exenatide administration in an obese mouse model reversed hepatic steatosis. This effect was attributed to increased fatty acid oxidation and/ or inhibition of de novo lipogenesis (6). At present, it is not clear whether incretin mimetics also affect liver fat content in obese type 2 diabetes patients. 


\section{Case report}

A 59-year-old Caucasian male with type 2 diabetes, treated with metformin, was prescribed exenatide $20 \mu \mathrm{g}$ twice daily, because of poor control $\left(\mathrm{HbA}_{1} \mathrm{c}: 8.7 \%\right.$; reference values: 4-6\%). He was a retired craftsman and had no history of alcohol abuse or viral hepatitis. At baseline, his body weight was $88.5 \mathrm{~kg}$ (BMI $28.7 \mathrm{~kg} / \mathrm{m}^{2}$ ), his waist circumference was $98.5 \mathrm{~cm}$ and his blood pressure was $157 / 87 \mathrm{mmHg}$. The fasting laboratory values were as follows: plasma glucose $14.5 \mathrm{mmol} / 1$, total cholesterol $4.82 \mathrm{mmol} / 1$, HDL cholesterol $1.04 \mathrm{mmol} / 1$, LDL cholesterol $3.29 \mathrm{mmol} / 1$, triglcyerides $1.46 \mathrm{mmol} / 1$, alanine aminotransferase (ALT) 46 IU/1, aspartate aminotransferase (AST) $18 \mathrm{IU} / 1, \gamma$-glutamyltranspeptidase (GGT) $28 \mathrm{IU} / 1$ (reference values: 6-48; 10-45; 7-51 IU/1, respectively) and insulin resistance estimated by homeostasis assessment model (HOMA-IR) of 6.36 .

Following 44 weeks of exenatide therapy, $\mathrm{HbA}_{1} \mathrm{c}$ decreased to $8.4 \%$ and fasting plasma glucose to $9.9 \mathrm{mmol} / \mathrm{l}$. His body weight fell by $4.7 \%$, from 88.5 to $84.3 \mathrm{~kg}$, and his waist circumference by $2.5 \mathrm{~cm}$.

Liver fat accumulation was quantified in the fasting state using proton magnetic resonance spectroscopy ( $\left.{ }^{1} \mathrm{H}-\mathrm{MRS}\right)$, a method that has been shown to correlate excellently with liver fat as measured in biopsy samples (7). At three positions (right anterior, right posterior and medial or left anterior), a $15 \mathrm{~cm}^{3}$ spectroscopic volume of interest was positioned, avoiding major blood vessels, intra-hepatic bile ducts and the lateral margin of the liver. Areas of resonances from protons of water and methyl and methylene groups in fatty acid chains of the hepatic triglycerides were evaluated with LCModel (8). Surprisingly, the mean liver fat (average of the three volumes of interest) declined from 15.8\% before to $4.3 \%$, i.e. by $73 \%$, after 44 weeks of exenatide therapy (Fig. 1). This dramatic decrease in liver fat was accompanied by significant beneficial changes in several cardiovascular disease risk factors: blood pressure decreased to $140 / 85 \mathrm{mmHg}$, fasting LDL cholesterol to $2.67 \mathrm{mmol} / 1$, triglycerides to $0.69 \mathrm{mmol} / 1$, HOMA-IR to 2.51, while HDL cholesterol increased to $1.27 \mathrm{mmol} / 1$. In accordance with the MRS findings, all liver enzymes, and in particular ALT, the most important marker of liver steatosis (9), improved. This value decreased from 46 to $20 \mathrm{IU} / 1$, while AST decreased from 18 to $13 \mathrm{IU} / 1$, and GGT from 28 to $23 \mathrm{IU} / 1$.

\section{Discussion}

Experimental evidence indicates that exenatide, by activating the GLP-1 receptors, effectively
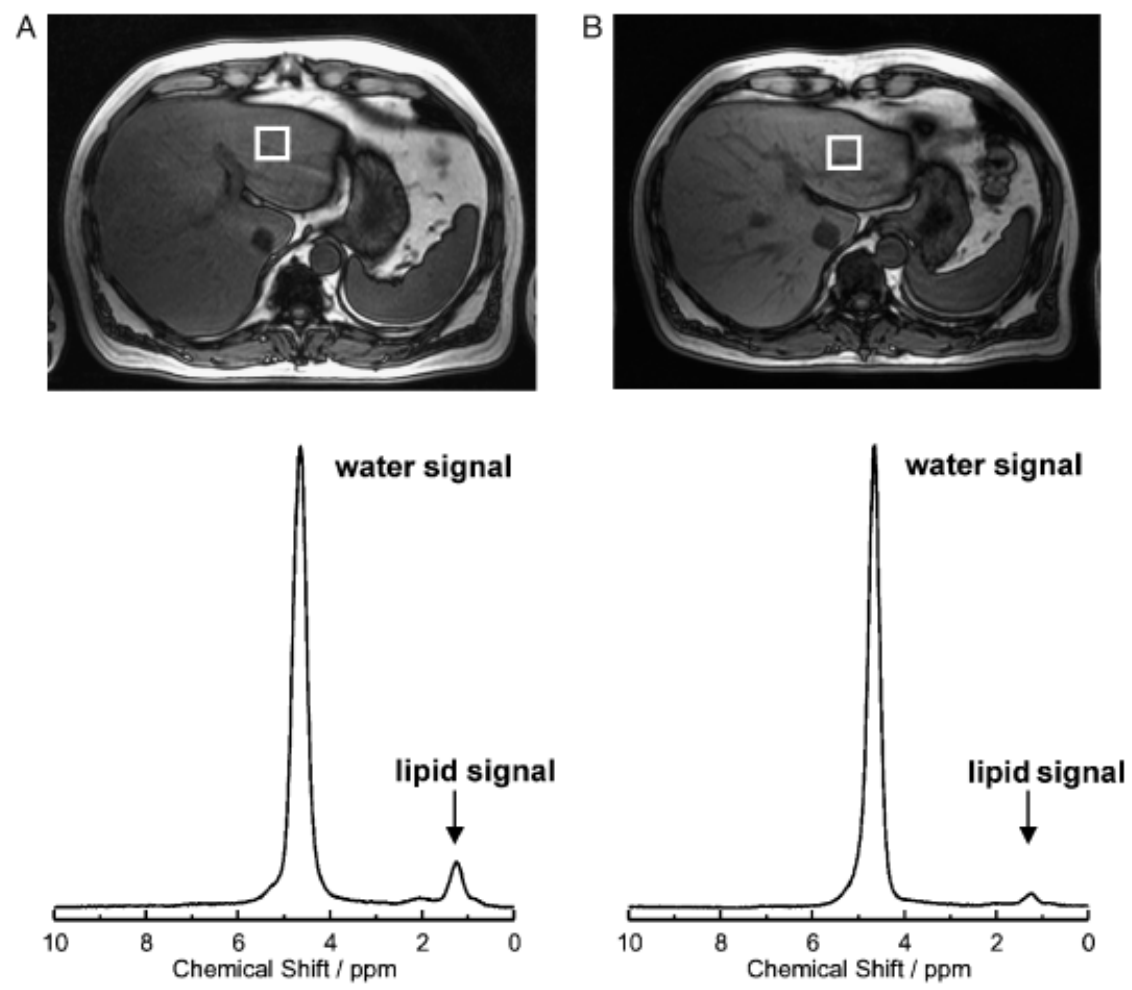

Fig. 1. Abdomen magnetic resonance imaging scans indicating volume of interest in the anterior left hepatic lobe from which proton magnetic resonance spectra $\left(\mathrm{CH}_{2}\right.$ peak at 1.3 p.p.m. is the main signal of lipids) were obtained before (A) and after (B) exenatide treatment. 
reverses hepatic steatosis. This is the first report describing a marked reduction in liver fat accumulation following treatment with the incretin mimetic exenatide in humans. Although this effect may be indirect, because of concomitant systemic metabolic improvement, it is very likely that exenatide may also act directly on the liver, as a GLP-1 receptor is present on liver cells (6). A direct effect is even more likely in the present case, as additional exenatide treatment in our patient did not lead to significant improvement of glycaemia, nor did it result in relevant weight loss, both of which are treatment effects that are known to reduce liver fat $(3,4)$. In vitro studies in hepatocytes demonstrated that exposure to synthetic exendin-4 resulted in an up-regulation of PPAR $\alpha$ mRNA, a key element in $\beta$-oxidation of free fatty acids, and in a down-regulation of sterol regulatory element binding protein (SREBP-1c) and stearoyl-CoA desaturase (SCD1), both critical regulators of de novo hepatic lipogenesis. As exenatide stimulates insulin secretion, and in turn inhibits lipolysis, the delivery of FFA to the liver will be diminished. This probably also contributes to the lowering of liver fat content.

Incretin mimetics have already been demonstrated to influence beneficially a variety of abnormalities in type 2 diabetes, including defective insulin secretion, hyperglucagonaemia as well as excessive body weight and appetite (5). Now it seems that incretin mimetics also have salient effects on NAFLD and the associated cardiovas- cular risk factors. Future long-term intervention studies are required to confirm these findings.

\section{References}

1. Dekker J M, Girman C, Rhodes T, et al. Metabolic syndrome and 10-year cardiovascular disease risk in the hoorn study. Circulation 2005; 112: 666-73.

2. Den Boer M, Voshol P J, Kuipers F, Havekes L M, Romisn J A. Hepatic steatosis: a mediator of the metabolic syndrome. Lessons from animal models. Arterioscler Thromb Vasc Biol 2004; 24: 644-9.

3. Tiikmainen M, Bergholm R, Vehravanara S, et al. Effect of identical weight loss on body composition and features of insulin resistance in obese women with high and low liver fat content. Diabetes 2003; 52: 701-7.

4. Bajaj M, Suraamornkul S, Pratipanawatr T, et al. Pioglitazone reduces hepatic fat content and augments splanchnic glucose uptake in patients with type 2 diabetes. Diabetes 2003; 52: 1364-70.

5. Riddle M C, Drucker D J. Emerging therapies mimicking the effects of amylin and glucagon-like peptide 1. Diabetes Care 2006; 29: 435-9.

6. Ding X, Saxena N K, Lin S, Gupta N, Anania F A. Exendin-4, a glucagon-like protein-1 (GLP-1) receptor agonist, reverses hepatic steatosis in ob/ob mice. Hepatology 2006; 43: 173-81.

7. Thomsen C, Becker U, Winkler K, Christoffersen P, Jensen M, Henriksen O. Quantification of liver fat using magnetic resonance spectroscopy. Magn Reson Imaging 1994; 12: 487-95.

8. Provencher S W. Estimation of metabolite concentrations from localized in vivo proton NMR spectra. Magn Reson Med 1993; 30: 672-9.

9. Marchesini G, Brizi M, Bianchi G, et al. Nonalcoholic fatty liver disease: a feature of the metabolic syndrome. Diabetes 2001; 50: 1844-50. 